\title{
Structure-function relationships of the polypyrimidine tract binding protein
}

\section{Journal Article}

\section{Author(s):}

Auweter, Sigrid D.; Allain, Frédéric H.-T.

Publication date:

2008-02

Permanent link:

https://doi.org/10.3929/ethz-b-000004851

\section{Rights / license:}

In Copyright - Non-Commercial Use Permitted

\section{Originally published in:}

Cellular and Molecular Life Sciences 65(4), https://doi.org/10.1007/s00018-007-7378-2 


\title{
Review
}

\section{Structure-function relationships of the polypyrimidine tract binding protein}

\author{
S. D. Auweter ${ }^{+}$and F. H.-T. Allain* \\ Institute for Molecular Biology and Biophysics, ETH Zürich, 8093 Zürich (Switzerland), Fax: + 41-44-633-1294, \\ e-mail: allain@mol.biol.ethz.ch
}

Received 16 August 2007; received after revision 18 September 2007; accepted 2 October 2007

Online First 3 November 2007

\begin{abstract}
The polypyrimidine tract binding protein (PTB) is a 58-kDa RNA binding protein involved in multiple aspects of mRNA metabolism including splicing regulation, polyadenylation, $3^{\prime}$ end formation, internal ribosomal entry site-mediated translation, RNA localization and stability. PTB contains four RNA recognition motifs (RRMs) separated by three linkers. In this review we summarize structural information on PTB in solution that has been gathered
\end{abstract}

during the past 7 years using NMR spectroscopy and small-angle X-ray scattering. The structures of all RRMs of PTB in their free state and in complex with short pyrimidine tracts, as well as a structural model of PTB RRM2 in complex with a peptide, revealed unusual structural features that provided new insights into the mechanisms of action of PTB in the different processes of RNA metabolism and in particular splicing regulation.

Keywords. Alternative splicing, translation regulation, polypyrimidine tract binding protein, RNA-protein complex, RNA-protein recognition, RNA recognition motifs, RBD, splicing regulation.

\section{The many functions of polypyrimidine tract binding protein}

The polypyrimidine tract binding protein (PTB), also referred to as hnRNP I, is an RNA binding protein of $58 \mathrm{kDa}$ shown to be involved in many different aspects of RNA metabolism and, as its name indicates, to bind preferentially poly-pyrimidine stretches $[1,2]$.

The most intensively studied function of PTB is its role as a regulator of alternative splicing (reviewed in [3-5]). In alternative splicing regulation, PTB binds to splicing silencer elements within the pre-mRNA (Fig. 1) and has been shown to be responsible for repression (i.e., exclusion) of many tissue-specific

Present address: Michael Smith Laboratories, University of British Columbia, Vancouver, BC V6T 1Z4, Canada.

* Corresponding author. exons in vertebrates including its own mRNA [1, 4, 6-36]. PTB can repress different classes of alternative exons, namely cassette exons (Fig. 1a,d), mutually exclusive exons (Fig. 1b) and alternative 3 'terminal exons (Fig. 1c). The distribution of PTB binding sites within introns has led to several proposed models of PTB action. For example, PTB could interfere with spliceosome assembly by binding to the branch point pyrimidine tract and therefore directly sequestering the branch-point or competing with U2AF, an essential component of the constitutive splicing machinery $[2,15,22,32]$. In most cases, however, the exons silenced by PTB are flanked by PTB binding sites on both adjacent introns, and mutations of upstream PTB binding sequences have been shown to reduce binding of PTB to the downstream sites [7]. Silencing of PTB-regulated exons was hence postulated to be due to the creation of a zone of 
a c-src mRNA

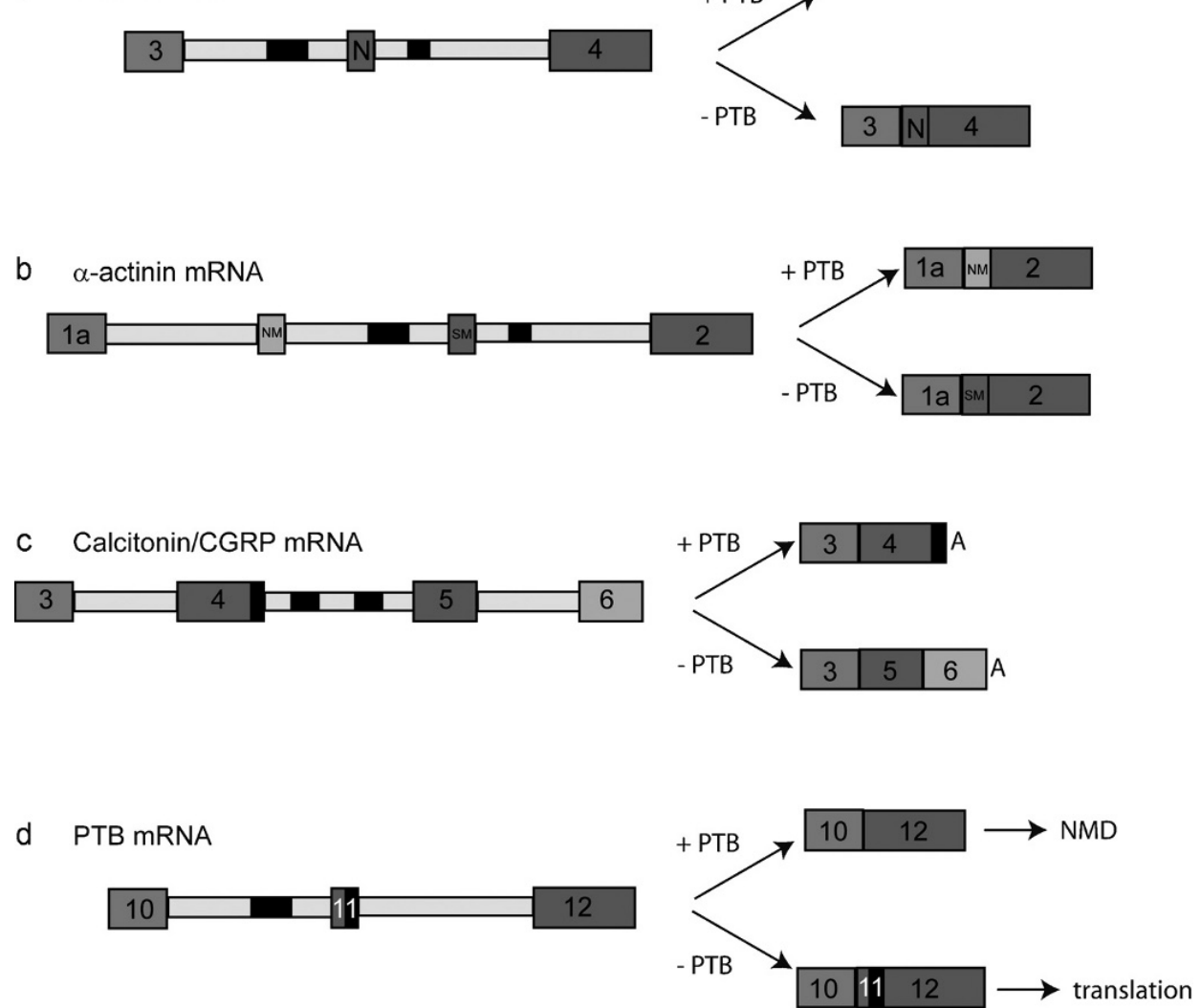

Figure 1. Polypyrimidine tract binding protein (PTB) is a ubiquitous regulator of alternative splicing that influences different types of alternative-splicing events $(a)$ PTB represses the N1 "cassette-exon" in the c-src pre-mRNA [7, 9, 10,103]. (b) PTB represses the "mutually exclusive" SM exon of the $\alpha$-actinin mRNA [12,13]. (c) PTB regulates the choice of the 3 -terminal exon of the calcitonin/CGRP mRNA [104]. (d) PTB autoregulates its own splicing and its mRNA level by repressing the inclusion of its own exon 11 . Skipping of exon 11 of PTB mRNA leads to non-sense-mediated decay (NMD) [16]. Black boxes indicate the location of pyrimidine tracts bound by PTB. Intronic sequences are shown in yellow, while colored boxes indicate exonic sequences. Arrows indicates splicing in the presence $(+)$ or absence $(-)$ of PTB.

silencing across the exon, either by oligomerization of PTB across the entire exon or by multimerization of upstream and downstream PTB to introduce a loop in the RNA [3]. More recently, it was found that PTB can act more indirectly, at early stages of spliceosome assembly, by preventing the establishment of productive interactions between U2AF and the U1 snRNP. This is mediated by PTB binding to intronic [11] or exonic sequences [27] that prevent intron or exon definition, respectively.

PTB is widely expressed across all developmental stages and cell types, and often represses the splicing of strictly tissue-specific exons outside of this particular tissue $[5,37]$. Hence, mechanisms that release PTB are important for tissue-specific regulation of splicing. Another facet of splicing regulation by PTB is the existence of co-repressors like Raver1 [38, 39]. Raver1 recruitment is essential for effective repressor function of PTB in certain pre-mRNAs, for example for the splicing repression of $\alpha$-tropomyosin exon 3 , while in other systems PTB alone is sufficient. Furthermore, tissue-specific paralogs of PTB exist, such as neuronally enriched PTB $[9,40]$ (nPTB), the smooth muscle-specific variant (smPTB) [41] or ROD1 the variant found in rat hematopoietic cell [42], which appear to have different effects on splicing regulation $[9,41]$.

The second most studied function of PTB is its role in internal ribosomal entry site (IRES)-mediated translation initiation of both cellular and viral mRNAs. IRESs are large RNA structures present in the 5'untranslated regions (UTR) of some cellular mRNAs $[43,44]$ and of many viral mRNAs [45] (Fig. 2). IRESs help recruiting the translation machinery to initiate translation. This recruitment often requires cellular factors binding the IRES (initiation of translation accessory factors, ITAFs) for efficient translation initiation [43, 44, 46, 47]. PTB is one of the most frequently encountered ITAFs and was found to be implicated in IRES-mediated translation of several 
a

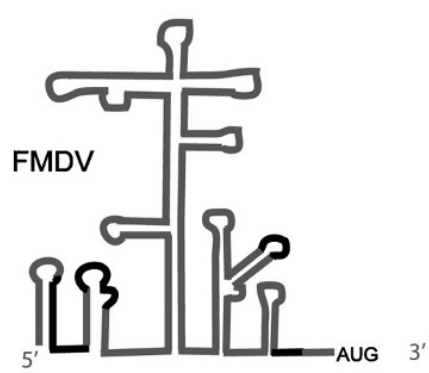

C

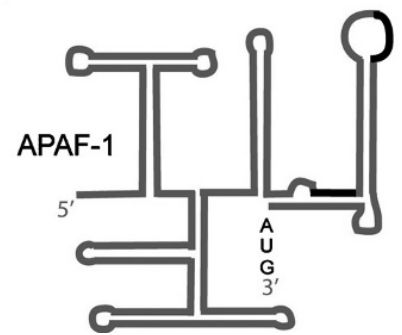

b

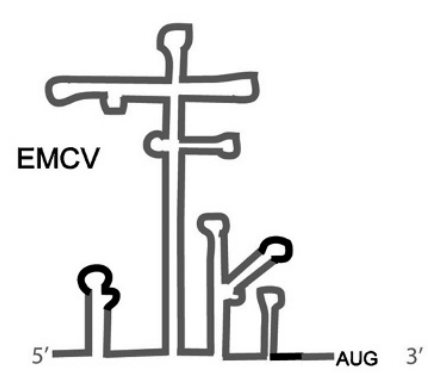

$3^{\prime}$

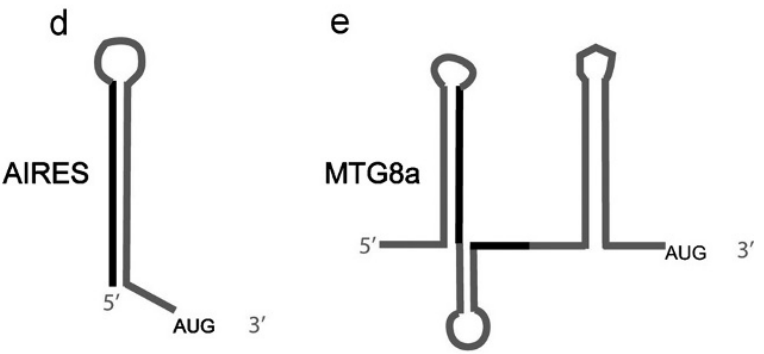

Figure 2. PTB regulates internal ribosomal entry site (IRES)-mediated translation initiation of many viral and cellular mRNAs. The secondary structures of two viral IRESs are shown: (a) FMDV (foot and mouse disease virus) [50] and (b) EMCV (encephalomyocarditis virus) [50]. The secondary structures of three cellular IRESs are shown: $(c)$ APAF-1 (apoptosis protease activating factor 1) [56], $(d)$ Artificial IRES [53] and (e) MTG8a [53]. Black lines indicate the locations of the pyrimidine tracts bound by PTB.

human viruses including hepatitis viruses $\mathrm{A}$ and $\mathrm{C}$ (although this is controversial $[48,49])$ and several picornavirus (aphtoviruses and cardioviruses, Fig. 2). Several footprinting studies have mapped PTB binding sites on different IRESs and identified several pyrimidine stretches embedded in stem loops and single-stranded regions (in domain $\mathrm{K}$ and $\mathrm{H}$ of the picornaviruses type II IRESs [50, 51], in domain 3 of the hepatitis $\mathrm{C}$ virus [52] and also in cellular RNAs [53-55], see Fig. 2). Moreover, reports have shown that interactions between PTB (or the neuronal nPTB) and cellular [56] or viral IRESs [51] are essential for the IRESs to attain their correct functional conformation. Hence, in its interaction with IRES RNAs, PTB acts as an RNA chaperone. Although in most cases PTB positively regulates IRES-mediated translation of cellular RNA [55-59], PTB can also be a negative regulator, like with the IRES of the UNR protein (upstream of N-ras), another ITAF [60], or of the bip mRNA [61]. More recently, PTB was also found associated with the $3^{\prime}$ UTR of the ATP synthase $\beta$-subunit, where it helps enhancing translation in a cap-independent manner [62].

In addition to its role in splicing and translation regulation, PTB is implicated in $3^{\prime}$-end processing [63, 64], localization [65, 66], and stability [67-75] of various cellular mRNAs. In these systems, PTB acts through pyrimidine-tract binding sites within $5^{\prime}$ and/or $3^{\prime}$ UTRs. Finally, recent genome-wide investigations
[76] have identified the association of PTB with a distinct subset of cellular mRNAs that encode proteins implicated in cellular transport, vesicle trafficking and apoptosis [77], suggesting even more regulatory functions for PTB in the cell.

\section{The structure of PTB in its free state}

Human PTB has a molecular mass of about $58 \mathrm{kDa}$. It consists of four RNA recognition motifs of the RRM/ RBD/RNP type (RNA recognition motif/RNA binding domain/ribonucleoprotein) that are connected by linkers, plus an N-terminal nuclear localization signal (NLS) sequence (Fig. 3a). RRMs typically have a size of about 90 amino acid residues that fold in a $\beta \alpha \beta \beta \alpha \beta$ topology, where a four-stranded $\beta$-sheet packs against the two $\alpha$-helices [78]. In terms of primary sequence, RRMs are characterized by two conserved amino acid stretches termed RNP2 ([I/L/V]-[F/Y]-[I/L/V]-X-N$\mathrm{L})$ and RNP1 ([R/K]-G-[F/Y]-[G/A]-[F/Y]-[I/L/V]$\mathrm{X}-[\mathrm{F} / \mathrm{Y}])$. Interestingly, although the RRM domains of PTB could be identified in sequence homology searches, they match very poorly to this consensus (Fig. 3a). In particular, they lack most of the aromatic residues. In other RRM-containing proteins, however, these aromatic side chains have been shown to be crucial for both folding of the domain and RNA recognition, as one of them makes up part of the hydrophobic core of the domain, while others, in 
particular the aromatics at position 2 of RNP2 and positions 3 and 5 of RNP1, are commonly found to make direct contacts to RNA bases. These aromatic side chains are situated on the surface-exposed side of the structurally central $\beta 1$ and $\beta 3$ strands and provide a hydrophobic surface ideal for accommodating the bases of single-stranded nucleic acid molecules [78]. The apparent absence of these critical features in the primary sequence of PTB (Fig. 3a) has initially raised the interest in atomic resolution structures of PTB RRMs (Fig. 3b-e). The earliest of these studies describes the nuclear magnetic resonance (NMR) structure of a construct containing RRMs 3 and 4 [79]. This study revealed that despite the poor match with the RNP consensus, both domains fold in the canonical $\beta \alpha \beta \beta \alpha \beta$ RRM topology. In addition, quite unexpectedly, RRM3 features a large structured Cterminal extension that folds into an additional fifth $\beta$ strand (Fig. 3e). This strand lies anti-parallel to $\beta 2$ on one side of the $\beta$-sheet and is connected to $\beta 4$ on the opposite side by a long loop that stretches across the entire sheet. In this structure, which was solved in the absence of an RNA target, this linker seems to be fairly mobile and does not appear to make any contacts with the rest of the domain. Heteronuclear ${ }^{1} \mathrm{H}-{ }^{15} \mathrm{~N}$ NOE experiments confirmed that this linker undergoes fast internal motion in the sub-nanosecond time scale [79]. Both domains are characterized by a densely packed hydrophobic core and a mutational analysis shows that amino acids at those $\beta$-sheet positions that are usually occupied by aromatic side chains that stack with RNA bases, are important for RNA binding in PTB RRMs 3 and 4 as well, despite their non-aromatic nature [79]. Initially, RRM3 and 4 of PTB were suggested to be independent based on the lack of interdomain NOEs between the domains [79]. However, a subsequent structural characterization of the same construct revealed that this was not true [80]. In this second NMR study, segmental isotope labeling of the two domains was employed to obtain a large number of inter-domain distance constraints and demonstrate unambiguously the extensive interdomain interaction [80] (Fig. 3f). Measurements of protein dynamics, as well as a mutational analysis, could further confirm the interaction. As many as 20 side chains that lie in both helices of RRM3, in helix 2 of RRM4 and in the interdomain linker form a hydrophobic cluster that glues the two domains together so that their RNA binding interfaces point in opposite directions [80]. This large interaction between two RRMs is very unusual among RRMs in their free state, the only other example being the recent structure of Prp24, a protein containing three RRMs [81]. The amino acids contributing to the unusual inter-domain interactions between RRM3 and RRM4 are highly conserved across species, as well as in PTB paralogs $[41,42]$ like nPTB $[9,40]$ and hence it seems likely that this topology is functionally significant.

The NMR structures of free RRM1 and RRM2 have been assessed in another study [82]. This analysis revealed that RRM1 folds as a canonical $\beta \alpha \beta \beta \alpha \beta$ RRM (Fig. 3b). Interestingly, the $C$ terminus of this domain binds across the $\beta$-sheet in a conformation that is stabilized by numerous hydrophobic contacts, suggesting that the $\beta$-sheet might be incapable of binding RNA. Nevertheless, chemical shift mapping experiments revealed that RRM1 uses the canonical $\beta$-sheet binding surface for the recognition of RNA ligands [82]. It remained unclear, however, whether a conformational change, i.e., the displacement of the $\mathrm{C}$ terminus from the sheet, was necessary for RNA binding. RRM2, on the other hand, has a tertiary structure resembling RRM3, with the domain being extended by a fifth $\beta$-strand (Fig. 3c). This additional $\beta$-strand seems to be stabilized in both RRMs by a stacking interaction between a tyrosine in $\beta 5$ (Y275 in RRM2 and Y430 in RRM3), and a histidine (H201 in RRM2) or a phenylalanine (F365 in RRM3) from $\alpha$ helix1 (Fig. 3c, e). In the case of RRM2, however, the $\beta 4-\beta 5$ linker is fairly short and rigid and makes close contacts to the $\beta$-sheet. Chemical shift mapping of this domain furthermore shows that the C-terminal extension and $\beta 5$ both participate in RNA binding [82]. However, all NMR structures of PTB were solved using either single domains or tandem domain constructs and it remained unclear how full-length PTB behaves in solution. Are RRM1, RRM2 and RRM34 really fully independent, or is there a preferred relative orientation of the domains? Small angle Xray scattering experiments with full-length PTB and different PTB domain constructs provided low resolution structures of the entire protein and hence insight into these questions [82, 83]. These studies confirmed that the RRM34 construct adopts a compact, globular structure and revealed a more or less linear arrangement of the four RRM domains, resulting in an elongated particle. Furthermore, it appears that there is reduced conformational flexibility between RRMs 2 and 3; probably not caused by direct contacts between the domains, but possibly due to transiently structured elements within the inter-domain linker [83]. Interestingly, this linker is subject to alternative splicing and hence its behavior in solution is likely to be relevant for proper PTB function. Finally, these studies, as well as others, could unambiguously show that full-length PTB is a monomer in solution $[10,51,84]$. The possibility remains, however, that binding of certain RNA targets might facilitate PTB-PTB interactions. 

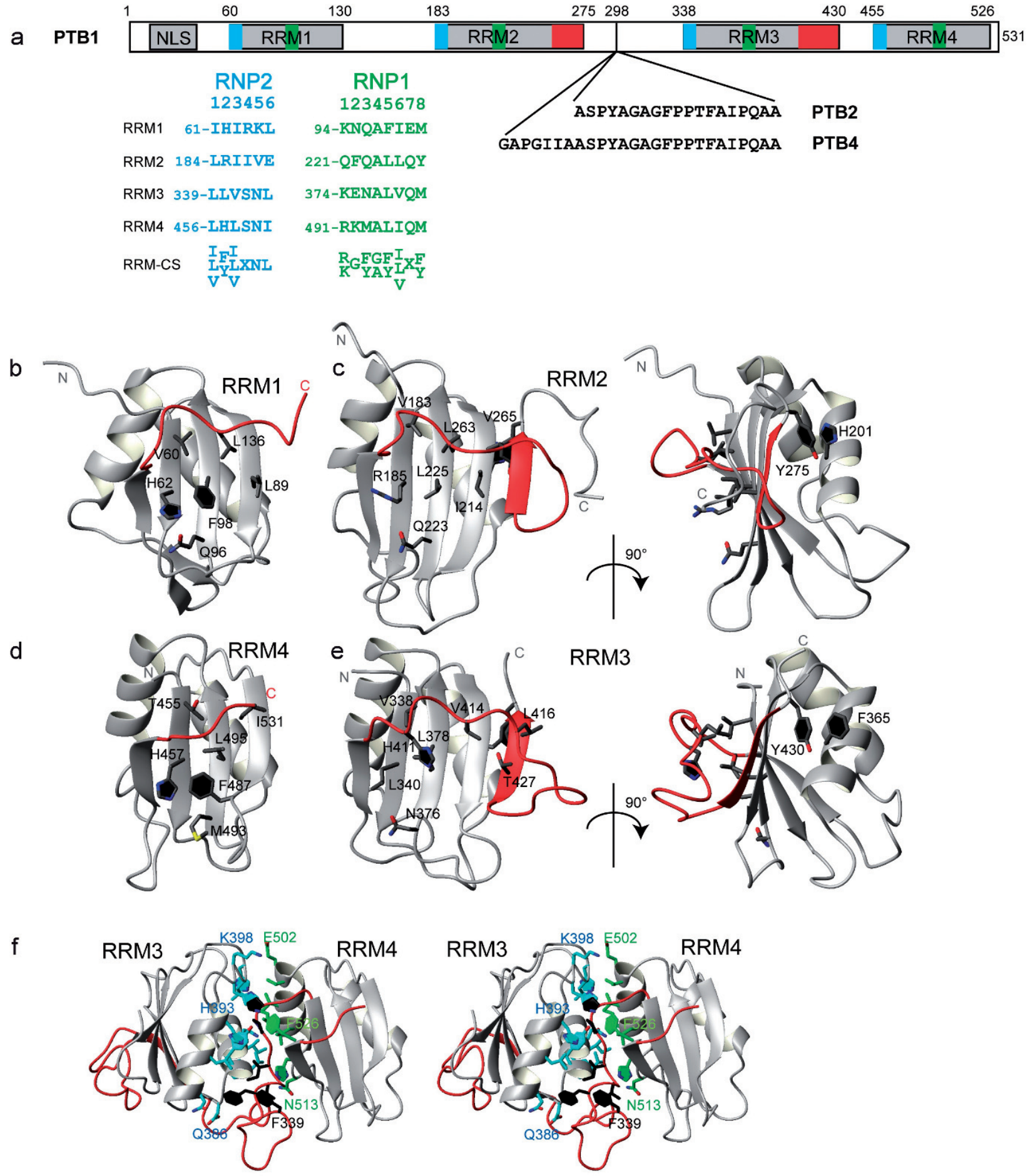

Figure 3. The structure of the free PTB. (a) Domain composition of PTB1. The gray, blue, green and red boxes indicate the location of the RNA recognition motifs (RRMs), the RNP2 and RNP1 sequences, and the extended domain comprising the additional $\beta 5$ strand, respectively. The amino acid sequences of RNP1 and RNP2 of each RRM and of a consensus RRM (RRM-CS) are shown as well as the amino acid sequences of the two other isoforms of PTB, PTB2 and PTB4. Structures of RRM1 [82] $(b)$, RRM2 [82] $(c)$, RRM4 [79, 80] $(d)$ and RRM3 $[79,80](e)$ in their free form. The ribbons of the RRMs are shown in gray and the ribbons of the C-terminal extensions are shown in red. Side-chains of important residues are shown in gray. $(f)$ Stereoview of the interacting domains RRM3 and RRM4 in the free form [80]. The ribbons of the RRMs are shown in gray and the ribbons of the C-terminal extensions and the interdomain linker are shown in red. Protein side-chains contributing to the interdomain interface are shown in blue (RRM3), green (RRM4) and black (linker), respectively. 
Hence, despite the discrepancy between consensus RRMs and PTB RRMs in the primary sequence, the structures of the RRMs of PTB revealed that residues at equivalent positions within the RNP sequences appeared to fulfill equivalent functions in RRM folding and RNA binding. However, a few open questions remained. How exactly do the $\beta$-sheets recognize RNA in the absence of aromatic side chains that serve as stacking partners, and how do the additional $\beta$-strands of RRM2 and RRM3 assist in RNA recognition? Does each RRM have a defined sequence specificity? Will conformational changes occur upon RNA binding?

\section{The structure of PTB in complex with RNA and proteins}

To answer these questions, the NMR structures of all four RRMs of PTB were solved in complex with short pyrimidine tracts of the sequence 5'-CUCUCU-3' [85] (Fig. 4). This study could show that each RRM of PTB binds one RNA molecule of the sequence CUCUCU and could confirm that all the RRMs bind pyrimidine tracts with the help of their $\beta$-sheet $[82,86]$. RRM1 (Fig. 4a) and RRM4 (Fig. 4c) recognize three nucleotides (a UCU triplet), whereas RRM2 (Fig. 4b) recognizes a CUNU sequence ( $\mathrm{N}$ being any nucleotide) and RRM3 (Fig. 4d) binds the quintet UCUCU. Interestingly, in contrast to all other RRM-RNA complexes solved to date [87], the third $\beta$-strand, which contains the RNP1 residues, participates only weakly in RNA binding. As there are no aromatic residues at positions 3 and 5 of RNP1, hydrophobic residues in $\beta 2$ take over their function and stack with the RNA bases [85]. The C-terminal extensions, i.e., the additional $\beta$-strands, of RRM2 and RRM3 are indeed involved in RNA recognition (Fig. 4b, d). In the case of RRM2, one additional $3^{\prime} \mathrm{U}$ is bound, while RRM3 uses the extension to contact the $3^{\prime} \mathrm{CU}$ dinucleotide. In both cases, the linker between $\beta 4$ and $\beta 5$ makes most of these contacts [85]. A dense network of hydrogen bonds connects moieties of the protein backbone and side chains with the functional groups of the bases. Hence, PTB is a sequence-specific RNA binding protein with a different consensus sequence recognized by each domain. While the hydrogen bond network present in RRM1 predicts that any YCU sequence can be bound, RRM2, RRM3 and RRM4 specifically recognize CU(N)N, YCUNN and $\mathrm{YCN}$, respectively [85] (where $\mathrm{Y}$ stands for pyrimidine and $\mathrm{N}$ for any nucleotide). Finally, the overall fold of the PTB RRMs is identical in free [79, 80, 82] and RNA-bound forms [85] (Figs. 3 and 4). Solely some of the linkers appear to adopt a more ordered structure in the presence of RNA, in particular the linkers connecting $\beta 4$ and $\beta 5$ of RRM 2 and 3 . Interestingly, the structure of the RRM34 construct hardly changes upon RNA binding (Fig. 4e). Because of the unusual interaction between RRM3 and RRM4 that positions the two RNA binding surfaces away from each other (Fig. 4e), RRM34 binds two short pyrimidine tracts independently. Hence, NMR analyses revealed that, while RRMs 1 and 2 of PTB tumbled independently in solution, RRMs 3 and 4 form a single globular protein moiety, both in its free and RNA-bound forms [85].

As mentioned above, for the splicing repression of certain exons, PTB requires co-repressors like, for example, Raver1. Interestingly, Rideau et al. [88] could show by pulldown experiments and in vivo fluorescence resonance energy transfer (FRET) that several fragments of the Raver1 protein could bind to PTB. Mutational analyses and NMR titration experiments showed that a conserved sequence element ([S/ $\mathrm{G}]-[\mathrm{I} / \mathrm{L}]-\mathrm{L}-\mathrm{G}-\mathrm{X}-\mathrm{X}-\mathrm{P})$, which is present four times within the Raver1 sequence, is responsible for the interaction, and that the interaction is mediated by RRM2 of PTB. The authors could furthermore show that the interaction of the $[\mathrm{S} / \mathrm{G}]-[\mathrm{I} / \mathrm{L}]-\mathrm{L}-\mathrm{G}-\mathrm{X}-\mathrm{X}-\mathrm{P}$ element with PTB is essential for Raver1-mediated exon repression, but that additional elements, located in the $\mathrm{C}$ terminus of the Raver1 protein, are required for full repressor activity. NMR analyses with a Raver1 PGVSLLGAPPKD peptide revealed that it is the helical face of RRM2 that interacts with Raver1 (Fig. 4f) and that RRM2 can form a ternary complex in which a pyrimidine tract is bound to the $\beta$-sheet surface, while simultaneously the helical side serves as an interaction platform for the Raver1 peptide motif [88]. Thereby, the Raver1 motif binds in the shallow groove formed by $\alpha 1$ and the $\alpha 2 \beta 4$ loop of PTB RRM2, with the two leucines of the Raver1 peptide engaged in hydrophobic contacts [88] (Fig. 4f).

\section{How can the PTB structures explain its multiple functions}

The structural information gathered on the PTB protein has disclosed several new aspects of its function. The structures of the individual RRMs of PTB in complex with RNA have revealed the number and identity of the nucleotides bound by each RRM and the minimum linker length (more than 10 nucleotides) between two pyrimidine-tracts in order for both RRM3 and RRM4 to be bound to the RNA [85] (Fig. 4). This allows a more meaningful interpretation of experiments that aim at mapping PTB binding sites within longer RNA molecules known 

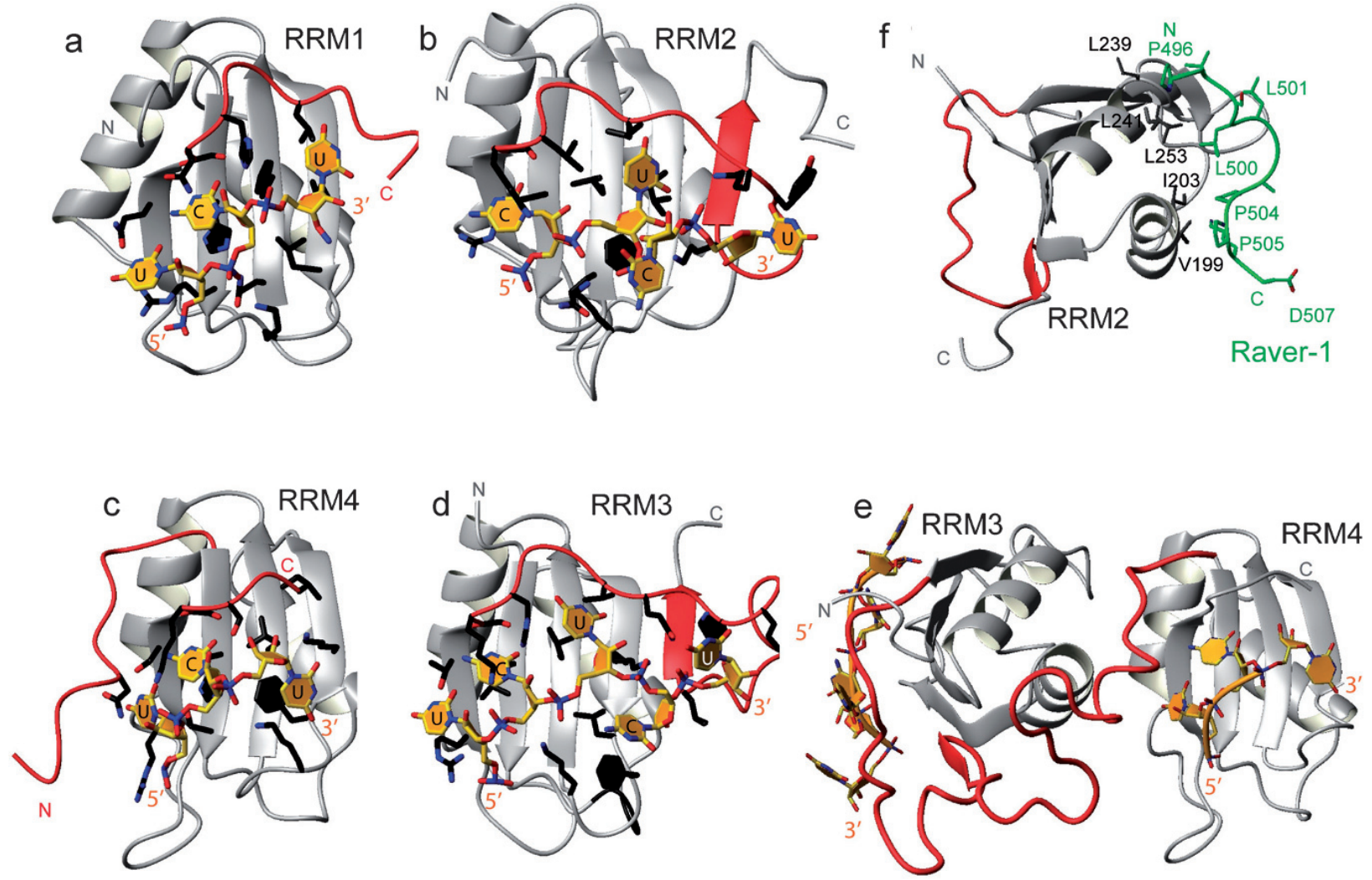

Figure 4. The structure of PTB bound to RNA and Raver1. Structures of PTB RRM1 $(a)$, RRM2 $(b)$, RRM4 $(c)$ and RRM3 $(d)$ bound to CUCUCU RNA [85]. (e) Structure of RRM34 in complex with two pyrimidine tracts [85]. ( $f$ ) Model of RRM2 bound to a peptide (P496D507) from Raver1 [88]. PTB side-chains involved in binding RNA are shown in black. RNA is shown in orange and Raver1 is shown in green.

to function through an interaction with $\mathrm{PTB}$, and might facilitate the identification of novel PTB targets. For example, many studies identified PTB binding sites by boundary analyses, gel shift experiments and cross-linking [10, 15, 22, 32]. In light of the complex structures [85], these findings can be more easily interpreted. For example the minimum binding sites for PTB RRM1-3 must contain at least 15 pyrimidines (considering the 12 nucleotides bound by the three RRMs and the necessary allowed spacing between each RRMs). This now explains that only two PTB binding sites, a high-affinity one spanning 20 nucleotides and a low affinity one spanning 12 nucleotides can be found on the $3^{\prime}$ splice-site of the c-src N1 exon [10]. While all three RRMs are likely to be accommodated on the 20-nucleotide site, only two RRMs could bind on the remaining RNA, which will lower the affinity for the second molecule.

Furthermore, in splicing regulation, it has been previously proposed [2] and more recently shown $[15,32]$ that PTB might function by displacing U2AF, a factor essential for the initiation of a successful splicing event, from the branch point pyrimidine tract. $\mathrm{U} 2 \mathrm{AF}$ has been shown to bind specifically to poly(U) tracts [2, 89]. In contrast, the structures of the PTB RRMs in complex with RNA show that PTB binds preferentially to pyrimidine tracts containing cytosines [85] (Fig. 4). Hence, the frequency of splicing of a certain exon might be regulated in the cell by varying the number of cytosines within the branch point pyrimidine tract, making it a higher affinity target for either of the two factors. Indeed, affinity measurements [90] and molecular dynamics simulations [91] could confirm that RRMs 2 and 3 of PTB bind poly(U) with significantly reduced affinity as compared to $\operatorname{poly}(\mathrm{CU})$.

One of the most interesting features in terms of the biological function of PTB that have been revealed by structural analyses is the prominent inter-domain contact between RRMs 3 and 4 [80, 85] (Figs. $3 f$ and 4e). Several structures of two tandem RRMs bound to RNA have been determined to date. In most of these cases, as, for example, the poly(A) binding protein (PABP) [92], sex-lethal [93], or nucleolin [94], both RRMs are separated by a small linker and bind adjacent stretches within the same RNA molecule. This topology provides a single, large RNA binding surface. In the case of PTB, however, the 

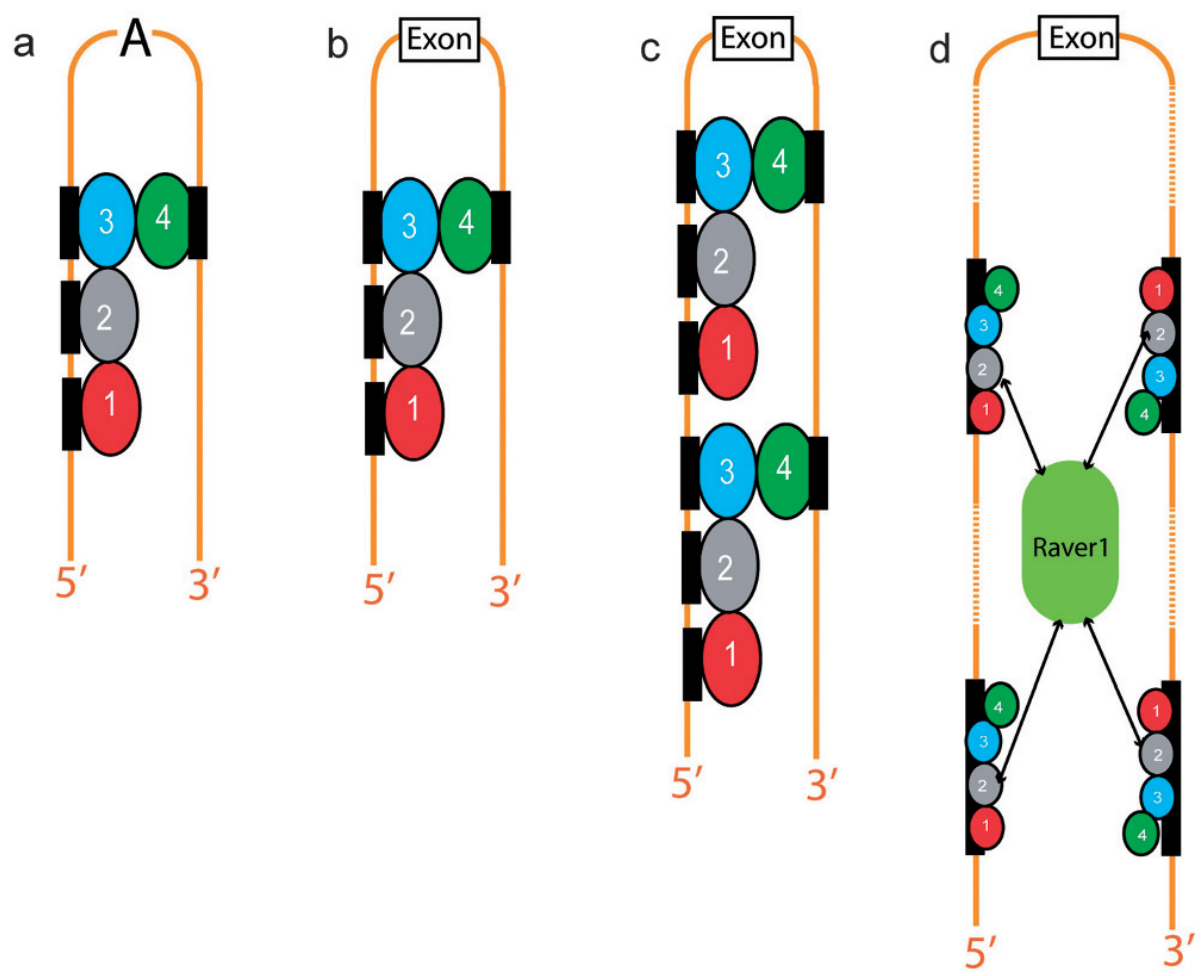

Figure 5. Mechanisms of splicing repression by PTB. (a) PTB could repress splicing by looping out either a branch-point adenine [22] or $(b)$ an alternative exon [25, 104]. (c) Model of cooperative binding around an alternative exon that requires binding of multiple PTB molecules [10]. (d) Model of how PTB and Raver1 can cooperate to loop out and therefore repress the splicing of an alternative exon that is flanked by distant intronic pyrimidine tracts [88].

relative orientation of tandem RRMs 3 and 4 makes it impossible for the two domains to bind immediately adjacent RNA sequences [85] (Fig. 4e). Instead, a linker of about 15 nucleotides between two pyrimidine tracts is necessary if these pyrimidine tracts are to be bound by both RRM3 and RRM4. Hence, this topology provides an ideal scaffold to induce RNA looping (Fig. 5). Therefore, the structure suggests that in contrast to the previous models mentioned above, PTB multimerization is not required for loop induction. Rather, a single molecule of PTB might fulfill this task with the help of its Cterminal RRM3 and RRM4 (Fig. 5a, b). In line with this model is the large body of evidence showing that PTB is indeed a monomer in solution [10, 51, 84], while previous measurements that found PTB is dimeric were most likely complicated by the fact that full-length PTB adopts an elongated shape, leading to an apparent larger size in native gels and sizeexclusion columns, and by the fact that under nonreducing conditions disulfide-mediated PTB dimers could form [84]. In other words, with the help of RRMs 3 and 4, PTB can bring pyrimidine tracts to close proximity that may be fairly far apart within the primary sequence of the RNA. This capacity might also explain many of the other functions of PTB, like its role in IRES-mediated translation initiation [43, $44,46,47]$. The structure of the complex also explains how several pyrimidine-rich pentaloops could create a PTB binding site in an IRES [50] or the 3'UTR of certain viral RNAs [95, 96], since all four RRMs bind only three to five pyrimidines each. However, it remains to be determined which of the four RRMs can bind such pentaloops (Fig. 2). Furthermore, the unusual topology of RRM3 and 4 suggests that PTB, by binding distant pyrimidine-tracts within the IRES, could induce a dramatic conformational change in the RNA, consistent with its role as an RNA chaperone [51, 56, 97]. Indeed, RRM 3 and 4 of PTB appear to be sufficient to stimulate the foot and mouse disease virus (FMDV) IRES-driven translation [51]. Finally, the unique conformation of PTB RRM34 explains how PTB might potentially bridge $3^{\prime}$ and $5^{\prime}$ UTRs as observed in certain viral $[98,99]$ or cellular [72] mRNAs. The RRM34 scaffold (Fig. 4e) appears to be an ideal tool for this RNA remodeling function.

It was demonstrated that more than one PTB molecule is necessary for splicing regulation $[10,15]$ and that PTB molecules cooperatively bind RNA $[10,15$, 100]. How could this multimerization take place without protein-protein interactions? In looping-out RNA, PTB binding brings distant pyrimidine tracts in close proximity, which could then favor binding of a second molecule of PTB (Fig. 5c) that could then favor binding of a third one, etc ... In this context, cooperative multimerization could be explained without the need for any PTB-PTB interaction. Hence, the mode of action of PTB as a splicing repressor could be explained by its capacity for looping out RNA [7] or 
for multimerizing to create a zone of silencing [15] as suggested previously [3] (Fig. 5).

The structural model of PTB RRM2 bound to the Raver1 peptide (Fig. 4f) suggests several possible modes of coordinated PTB and Raver1-mediated splicing repression [88]. First of all, binding of Raver1 to the helical side of RRM2 might bring PTB and Raver1 repressor domains in a correct relative orientation so that they can act in concert to regulate adjacent exons (Fig. 5d). These repressor domains might include the Raver1 C-terminus and the linker Cterminal to RRM2 of PTB, which have both been shown to be important for the repressor function of these proteins. Further evidence hinting at the functional importance of the RRM2-RRM3 linker of PTB includes the fact that it is alternatively spliced and that it seems to be partially structured. Secondly, Raver1 and PTB could cooperate to recruit additional factors. In particular, the $\mathrm{C}$ terminus of Raver1 contains PXXP and PPLP motifs that are known to serve as protein-protein interaction sites in other proteins. The RRM2-RRM3 linker of PTB might fulfill a similar role. Finally, as there are four PTB RRM2 recognition motifs of sequence $[\mathrm{S} / \mathrm{G}]-[\mathrm{I} / \mathrm{L}]-\mathrm{L}-\mathrm{G}-\mathrm{X}-\mathrm{X}-\mathrm{P}$ present in the Raver1 sequence, Raver1 might act as a bridging molecule (Fig. 5d), mediating contacts between different PTB molecules. In this context, it is interesting to speculate that Raver1 might be required for splicing repression in systems where upstream and downstream pyrimidine tracts recognized by PTB are quite far apart in primary sequence and hence PTB requires assistance for loop formation [88] (Fig. 5d). Indeed, in the strongly Raver1-dependent $\alpha$-tropomyosin exon 3 , PTB binding elements around the exon are about 460 nucleotides apart, whereas in the Raver1-independent $c$-src N1 exon, PTB binding sites are contained within just about 120 nucleotides.

\section{Conclusion and perspectives}

A large body of high- and low-resolution structural data on the PTB has been collected during the past 7 years. These studies have provided meaningful insights into the function of this versatile and ubiquitous protein. Recent studies have shown a role for PTB in ovarian cancer, suggesting that PTB could be a good potential therapeutic target [101]. These structures could, therefore, facilitate the design of a drug against this cancer. However, many open questions remain for future research.

First of all, it will be interesting to solve the structure of the PTB paralog nPTB. There is accumulating evidence that this protein, despite its high homology to PTB, has distinct functional properties. For exam- ple, splicing constructs, which appear to be solely regulated by PTB, have been shown to be more effectively spliced in neuronal cell extracts than in HeLa or kidney cell extracts. Hence, neuronal nPTB shows significantly reduced repressor function [9]. Furthermore, a recent micro-array study showed that PTB and nPTB have a mutually exclusive expression and regulate different sets of alternative exons during neuron development [30]. It will be most interesting to see whether the functional differences between PTB and $\mathrm{nPTB}$ are reflected in the $\mathrm{nPTB}$ structure.

Furthermore, all structural information has so far been derived with very short single-stranded pyrimidine oligonucleotides (Fig. 4). Recent convincing studies have shown that pyrimidine tracts embedded in the stem of a stem-loop are good binding sites for PTB within IRESs [53] (Fig. 2d, e). The present structure of PTB in complex with RNA [85] cannot explain how PTB could recognize such RNA stemloops, suggesting that PTB may bind these RNAs via a different mode of recognition and perhaps a different RNA binding surface. Moreover, structural studies using larger natural RNA targets would be crucial for a more complete understanding of PTB function, in particular a PTB-bound IRES RNA, where PTB makes simultaneous contacts to both single-strand and stem-loop RNA stretches (Fig. 2). These analyses could reveal the detailed role of PTB as an RNA remodeler of the IRESs [56].

Small angle X-ray scattering has revealed that the linker between RRMs 2 and 3 is partially structured [83]. Furthermore, there is evidence that this linker is important for the repressor function of PTB, and the fact that this linker is alternatively spliced is consistent with an important role of this part of the protein for PTB function. Therefore, it will be meaningful to examine its functional role, as well as its structure, in more detail and look for differences between the splice variants.

Beside U2AF, the repressive role of PTB has been antagonized by many different RNA binding proteins, namely the CELF $[13,25]$ proteins ETR3 and CUGBP, RBM4 [33], TIA-1 [27, 34], Nova [40] and Fox-1 [102]. It will be valuable to gain a better understanding of the molecular mechanisms behind these antagonisms.

Finally, it will be challenging to study the structures of higher order complexes containing full-length PTB, natural RNA targets and protein interaction partners. These studies could provide insights into the functional molecular machineries assembling on RNA molecules within the cell. 
Acknowledgment. The authors would like to thank Prof. Steve Matthews for providing several models of RRM2-Raver1 and Dr. Christophe Maris and Mr. Florian Oberstrass for critical reading of the manuscript. The authors acknowledge support from the SNFNCCR Structural Biology to FHTA.

1 Perez, I., Lin, C. H., McAfee, J. G. and Patton, J. G. (1997) Mutation of PTB binding sites causes misregulation of alternative 3' splice site selection in vivo. RNA 3, 764-778.

2 Singh, R., Valcarcel, J. and Green, M. R. (1995) Distinct binding specificities and functions of higher eukaryotic polypyrimidine tract-binding proteins. Science 268, 1173-1176.

3 Wagner, E. J. and Garcia-Blanco, M. A. (2001) Polypyrimidine tract binding protein antagonizes exon definition. Mol. Cell. Biol. 21, 3281-3288.

4 Spellman, R., Rideau, A., Matlin, A., Gooding, C., Robinson, F, McGlincy, N., Grellscheid, S. N., Southby, J., Wollerton, M. and Smith, C. W. (2005) Regulation of alternative splicing by PTB and associated factors. Biochem. Soc. Trans. 33, 457-460.

5 Valcarcel, J. and Gebauer, F. (1997) Post-transcriptional regulation: The dawn of PTB. Curr. Biol. 7, R705-R708.

6 Spellman, R., Llorian, M. and Smith, C. W. (2007) Crossregulation and functional redundancy between the splicing regulator PTB and its paralogs $\mathrm{nPTB}$ and ROD1. Mol. Cell 27, 420-434.

7 Chou, M. Y., Underwood, J. G., Nikolic, J., Luu, M. H. and Black, D. L. (2000) Multisite RNA binding and release of polypyrimidine tract binding protein during the regulation of c-src neural-specific splicing. Mol. Cell 5, 949-957.

8 Chan, R. C. and Black, D. L. (1997) Conserved intron elements repress splicing of a neuron-specific c-src exon in vitro. Mol. Cell. Biol. 17, 2970.

9 Markovtsov, V., Nikolic, J. M., Goldman, J. A., Turck, C. W., Chou, M. Y. and Black, D. L. (2000) Cooperative assembly of an hnRNP complex induced by a tissue-specific homolog of polypyrimidine tract binding protein. Mol. Cell. Biol. 20, 7463-7479.

10 Amir-Ahmady, B., Boutz, P. L., Markovtsov, V., Phillips, M. L. and Black, D. L. (2005) Exon repression by polypyrimidine tract binding protein. RNA 11, 699-716.

11 Sharma, S., Falick, A. M. and Black, D. L. (2005) Polypyrimidine tract binding protein blocks the 5 ' splice site-dependent assembly of U2AF and the prespliceosomal E complex. Mol. Cell 19, 485-496.

12 Southby, J., Gooding, C. and Smith, C. W. (1999) Polypyrimidine tract binding protein functions as a repressor to regulate alternative splicing of alpha-actinin mutually exclusive exons. Mol. Cell. Biol. 19, 2699-2711.

13 Gromak, N., Matlin, A. J., Cooper, T. A. and Smith, C. W. (2003) Antagonistic regulation of alpha-actinin alternative splicing by CELF proteins and polypyrimidine tract binding protein. RNA 9, 443-456.

14 Gooding, C., Roberts, G. C. and Smith, C. W. (1998) Role of an inhibitory pyrimidine element and polypyrimidine tract binding protein in repression of a regulated alpha-tropomyosin exon. RNA 4, 85-100.

15 Matlin, A. J., Southby, J., Gooding, C. and Smith, C. W. (2007) Repression of \{alpha\}-actinin SM exon splicing by assisted binding of PTB to the polypyrimidine tract. RNA 13, 1214 1223.

16 Wollerton, M. C., Gooding, C., Wagner, E. J., Garcia-Blanco, M. A. and Smith, C. W. (2004) Autoregulation of polypyrimidine tract binding protein by alternative splicing leading to nonsense-mediated decay. Mol. Cell 13, 91-100.

17 Gromak, N. and Smith, C. W. (2002) A splicing silencer that regulates smooth muscle specific alternative splicing is active in multiple cell types. Nucleic Acids Res. 30, 3548-3557.

18 Wollerton, M. C., Gooding, C., Robinson, F., Brown, E. C., Jackson, R. J. and Smith, C. W. (2001) Differential alternative splicing activity of isoforms of polypyrimidine tract binding protein (PTB). RNA 7, 819-832.
19 Carstens, R. P., Wagner, E. J. and Garcia-Blanco, M. A. (2000) An intronic splicing silencer causes skipping of the IIIb exon of fibroblast growth factor receptor 2 through involvement of polypyrimidine tract binding protein. Mol. Cell. Biol. 20, 7388-7400.

20 Wagner, E. J., Baraniak, A. P., Sessions, O. M., Mauger, D., Moskowitz, E. and Garcia-Blanco, M. A. (2005) Characterization of the intronic splicing silencers flanking FGFR2 exon IIIb. J. Biol. Chem. 280, 14017-14027.

21 Wagner, E. J. and Garcia-Blanco, M. A. (2002) RNAi-mediated PTB depletion leads to enhanced exon definition. Mol. Cell 10, 943-949.

22 Liu, H., Zhang, W., Reed, R. B., Liu, W. and Grabowski, P. J. (2002) Mutations in RRM4 uncouple the splicing repression and RNA-binding activities of polypyrimidine tract binding protein. RNA 8, 137-149.

23 Zhang, L., Liu, W. and Grabowski, P. J. (1999) Coordinate repression of a trio of neuron-specific splicing events by the splicing regulator PTB. RNA 5:117-130.

24 Ashiya, M. and Grabowski, P. J. (1997) A neuron-specific splicing switch mediated by an array of pre-mRNA repressor sites: evidence of a regulatory role for the polypyrimidine tract binding protein and a brain-specific PTB counterpart. RNA 3, 996-1015.

25 Charlet, B. N., Logan, P., Singh, G. and Cooper, T. A. (2002) Dynamic antagonism between ETR-3 and PTB regulates cell type-specific alternative splicing. Mol. Cell 9, 649-658.

26 Ladd, A. N., Stenberg, M. G., Swanson, M. S. and Cooper, T. A. (2005) Dynamic balance between activation and repression regulates pre-mRNA alternative splicing during heart development. Dev. Dyn. 233, 783-793.

27 Izquierdo, J. M., Majos, N., Bonnal, S., Martinez, C., Castelo, R., Guigo, R., Bilbao, D. and Valcarcel, J. (2005) Regulation of Fas alternative splicing by antagonistic effects of TIA- 1 and PTB on exon definition. Mol. Cell 19, 475-484.

28 Jin, W., Bruno, I. G., Xie, T. X., Sanger, L. J. and Cote, G. J. (2003) Polypyrimidine tract-binding protein down-regulates fibroblast growth factor receptor 1 alpha-exon inclusion. Cancer Res. 63, 6154-6157.

29 Cote, J., Dupuis, S. and Wu, J. Y. (2001) Polypyrimidine trackbinding protein binding downstream of caspase- 2 alternative exon 9 represses its inclusion. J. Biol. Chem. 276, 8535-8543.

30 Boutz, P. L., Stoilov, P., Li, Q., Lin, C. H., Chawla, G., Ostrow, K., Shiue, L., Ares, M. Jr. and Black, D. L. (2007) A posttranscriptional regulatory switch in polypyrimidine tractbinding proteins reprograms alternative splicing in developing neurons. Genes Dev. 21, 1636-1652.

31 Boutz, P. L., Chawla, G., Stoilov, P. and Black, D. L. (2007) MicroRNAs regulate the expression of the alternative splicing factor nPTB during muscle development. Genes Dev. 21, $71-84$.

32 Sauliere, J., Sureau, A., Expert-Bezancon, A. and Marie, J. (2006) The polypyrimidine tract binding protein (PTB) represses splicing of exon $6 \mathrm{~B}$ from the beta-tropomyosin pre-mRNA by directly interfering with the binding of the U2AF65 subunit. Mol. Cell. Biol. 26, 8755-8769.

33 Lin, J. C. and Tarn, W. Y. (2005) Exon selection in alphatropomyosin mRNA is regulated by the antagonistic action of RBM4 and PTB. Mol. Cell. Biol. 25, 10111-10121.

34 Shukla, S., Del Gatto-Konczak, F., Breathnach, R. and Fisher, S. A. (2005) Competition of PTB with TIA proteins for binding to a U-rich cis-element determines tissue-specific splicing of the myosin phosphatase targeting subunit 1 . RNA $11,1725-1736$.

35 Shen, H., Kan, J. L., Ghigna, C., Biamonti, G. and Green, M. R. (2004) A single polypyrimidine tract binding protein (PTB) binding site mediates splicing inhibition at mouse IgM exons M1 and M2. RNA 10, 787-794.

36 Le Guiner, C., Plet, A., Galiana, D., Gesnel, M. C., Del Gatto-Konczak, F. and Breathnach, R. (2001) Polypyrimidine tract-binding protein represses splicing of a fibroblast growth 
factor receptor- 2 gene alternative exon through exon sequences. J. Biol. Chem. 276, 43677-43687.

37 Smith, C. and Valcarcel, J. (2000) Alternative pre-mRNA splicing: the logic of combinatorial control. Trends Biochem. Sci. 25, 381-388.

38 Gromak, N., Rideau, A., Southby, J., Scadden, A. D., Gooding, C., Huttelmaier, S., Singer, R. H. and Smith, C. W. (2003) The PTB interacting protein raver1 regulates alpha-tropomyosin alternative splicing. EMBO J. 22, 6356-6364.

39 Huttelmaier, S., Illenberger, S., Grosheva, I., Rudiger, M., Singer, R. H. and Jockusch, B. M. (2001) Raver1, a dual compartment protein, is a ligand for PTB/hnRNPI and microfilament attachment proteins. J. Cell Biol. 155:775-786.

40 Polydorides, A. D., Okano, H. J., Yang, Y. Y., Stefani, G. and Darnell, R. B. (2000) A brain-enriched polypyrimidine tractbinding protein antagonizes the ability of Nova to regulate neuron-specific alternative splicing. Proc. Natl. Acad. Sci. USA 97, 6350-6355.

41 Gooding, C., Kemp, P. and Smith, C. W. (2003) A novel polypyrimidine tract-binding protein paralog expressed in smooth muscle cells. J. Biol. Chem. 278, 15201-15207.

42 Yamamoto, H., Tsukahara, K., Kanaoka, Y., Jinno, S. and Okayama, H. (1999) Isolation of a mammalian homologue of a fission yeast differentiation regulator. Mol. Cell. Biol. 19, 3829-3841.

43 Hellen, C. U. and Sarnow, P. (2001) Internal ribosome entry sites in eukaryotic mRNA molecules. Genes Dev. 15, 1593-1612.

44 Stoneley, M. and Willis, A. E. (2004) Cellular internal ribosome entry segments: Structures, trans-acting factors and regulation of gene expression. Oncogene 23, 3200-3207.

45 Jang, S. K. (2006) Internal initiation: IRES elements of picornaviruses and hepatitis c virus. Virus Res. 119, 2-15.

46 Belsham, G. J. and Sonenberg, N. (2000) Picornavirus RNA translation: Roles for cellular proteins. Trends Microbiol. 8, $330-335$

47 Spriggs, K. A., Bushell, M., Mitchell, S. A. and Willis, A. E. (2005) Internal ribosome entry segment-mediated translation during apoptosis: The role of IRES-trans-acting factors. Cell Death Differ. 12, 585-591

48 Brocard, M., Paulous, S., Komarova, A. V., Deveaux, V. and Kean, K. M. (2007) Evidence that PTB does not stimulate HCV IRES-driven translation. Virus Genes 35, 5-15.

49 Tischendorf, J. J., Beger, C., Korf, M., Manns, M. P. and Kruger, M. (2004) Polypyrimidine tract-binding protein (PTB) inhibits hepatitis $\mathrm{C}$ virus internal ribosome entry site (HCV IRES)-mediated translation, but does not affect HCV replication. Arch. Virol. 149, 1955-1970.

50 Kolupaeva, V. G., Hellen, C. U. and Shatsky, I. N. (1996) Structural analysis of the interaction of the pyrimidine tractbinding protein with the internal ribosomal entry site of encephalomyocarditis virus and foot-and-mouth disease virus RNAs. RNA 2, 1199-1212.

51 Song, Y., Tzima, E., Ochs, K., Bassili, G., Trusheim, H., Linder, M., Preissner, K. T. and Niepmann, M. (2005) Evidence for an RNA chaperone function of polypyrimidine tract-binding protein in picornavirus translation. RNA 11 $1809-1824$

52 Bock, R. and Maliga, P. (1995) In vivo testing of a tobacco plastid DNA segment for guide RNA function in psbL editing. Mol. Gen. Genet. 247, 439-443.

53 Mitchell, S. A., Spriggs, K. A., Bushell, M., Evans, J. R., Stoneley, M., Le, Quesne, J. P., Spriggs, R. V. and Willis, A. E. (2005) Identification of a motif that mediates polypyrimidine tract-binding protein-dependent internal ribosome entry. Genes Dev. 19, 1556-1571.

54 Spriggs, K. A., Mitchell, S. A. and Willis, A. E. (2005) Investigation of interactions of polypyrimidine tract-binding protein with artificial internal ribosome entry segments. Biochem. Soc. Trans. 33, 1483-1486.

55 Pickering, B. M., Mitchell, S. A., Spriggs, K. A., Stoneley, M and Willis, A.E. (2004) Bag-1 internal ribosome entry segment activity is promoted by structural changes mediated by poly(rC) binding protein 1 and recruitment of polypyrimidine tract binding protein 1. Mol. Cell. Biol. 24, 55955605.

56 Mitchell, S. A., Spriggs, K. A., Coldwell, M. J., Jackson, R. J. and Willis, A. E. (2003) The Apaf-1 internal ribosome entry segment attains the correct structural conformation for function via interactions with PTB and unr. Mol. Cell 11, $757-771$.

57 Schepens, B., Tinton, S. A., Bruynooghe, Y., Beyaert, R. and Cornelis, S. (2005) The polypyrimidine tract-binding protein stimulates HIF-1alpha IRES-mediated translation during hypoxia. Nucleic Acids Res. 33, 6884-6894.

58 Florez, P. M., Sessions, O. M., Wagner, E. J., Gromeier, M. and Garcia-Blanco, M. A. (2005) The polypyrimidine tract binding protein is required for efficient picornavirus gene expression and propagation. J. Virol. 79, 6172-6179.

59 Cho, S., Kim, J. H., Back, S. H. and Jang, S. K. (2005) Polypyrimidine tract-binding protein enhances the internal ribosomal entry site-dependent translation of p27Kip1 mRNA and modulates transition from G1 to S phase. Mol. Cell. Biol. 25, 1283-1297.

60 Cornelis, S., Tinton, S. A., Schepens, B., Bruynooghe, Y. and Beyaert, R. (2005) UNR translation can be driven by an IRES element that is negatively regulated by polypyrimidine tract binding protein. Nucleic Acids Res. 33, 3095-3108.

61 Kim, Y. K., Hahm, B. and Jang, S. K. (2000) Polypyrimidine tract-binding protein inhibits translation of bip mRNA. J. Mol. Biol. 304, 119-133.

62 Reyes, R. and Izquierdo, J. M. (2007) The RNA-binding protein PTB exerts translational control on 3'-untranslated region of the mRNA for the ATP synthase beta-subunit. Biochem. Biophys. Res. Commun. 357, 1107-1112.

63 Castelo-Branco, P., Furger, A., Wollerton, M., Smith, C., Moreira, A. and Proudfoot, N. (2004) Polypyrimidine tract binding protein modulates efficiency of polyadenylation. Mol. Cell. Biol. 24, 4174-4183.

64 Le Sommer, C., Lesimple, M., Mereau, A., Menoret, S., Allo, M. R. and Hardy, S. (2005) PTB regulates the processing of a 3 -terminal exon by repressing both splicing and polyadenylation. Mol. Cell. Biol. 25, 9595-9607.

65 Cote, C. A., Gautreau, D., Denegre, J. M., Kress, T. L., Terry, N. A. and Mowry, K. L. (1999) A Xenopus protein related to hnRNP I has a role in cytoplasmic RNA localization. Mol. Cell 4, 431-437.

66 Ma, S., Liu, G., Sun, Y. and Xie, J. (2007) Relocalization of the polypyrimidine tract-binding protein during PKA-induced neurite growth. Biochim. Biophys. Acta 1773, 912-923.

67 Tillmar, L. and Welsh, N. (2002) Hypoxia may increase rat insulin mRNA levels by promoting binding of the polypyrimidine tract-binding protein (PTB) to the pyrimidine-rich insulin mRNA 3'-untranslated region. Mol. Med. 8, 263-272.

68 Tillmar, L., Carlsson, C. and Welsh, N. (2002) Control of insulin mRNA stability in rat pancreatic islets. Regulatory role of a 3'-untranslated region pyrimidine-rich sequence. J. Biol. Chem. 277, 1099-1106.

69 Kosinski, P. A., Laughlin, J., Singh, K. and Covey, L. R. (2003) A complex containing polypyrimidine tract-binding protein is involved in regulating the stability of CD40 ligand (CD154) mRNA. J. Immunol. 170, 979-988.

70 Coles, L. S., Bartley, M. A., Bert, A., Hunter, J., Polyak, S., Diamond, P., Vadas, M. A. and Goodall, G. J. (2004) A multiprotein complex containing cold shock domain (Y-box) and polypyrimidine tract binding proteins forms on the vascular endothelial growth factor mRNA. Potential role in mRNA stabilization. Eur. J. Biochem. 271, 648-660.

71 Hamilton, B. J., Genin, A., Cron, R. Q. and Rigby, W. F. (2003) Delineation of a novel pathway that regulates CD154 (CD40 ligand) expression. Mol. Cell. Biol. 23, 510-525.

72 Knoch, K. P., Bergert, H., Borgonovo, B., Saeger, H. D., Altkruger, A., Verkade, P. and Solimena, M. (2004) Polypyrimidine tract-binding protein promotes insulin secretory granule biogenesis. Nat. Cell Biol. 6, 207-214. 
73 Pautz, A., Linker, K., Hubrich, T., Korhonen, R., Altenhofer, S. and Kleinert, H. (2006) The polypyrimidine tract-binding protein (PTB) is involved in the post-transcriptional regulation of human inducible nitric oxide synthase expression. J. Biol. Chem. 281, 32294-32302.

$74 \mathrm{Xu}, \mathrm{M}$. and Hecht, N. B. (2007) Polypyrimidine tract binding protein 2 stabilizes phosphoglycerate kinase 2 mRNA in murine male germ cells by binding to its 3'UTR. Biol. Reprod. 76, 1025-1033.

75 Kuwahata, M., Tomoe, Y., Harada, N., Amano, S., Segawa, H., Tatsumi, S., Ito, M., Oka, T. and Miyamoto, K. (2007) Characterization of the molecular mechanisms involved in the increased insulin secretion in rats with acute liver failure. Biochim. Biophys. Acta 1772, 60-65.

76 Gama-Carvalho, M., Barbosa-Morais, N. L., Brodsky, A. S., Silver, P. A. and Carmo-Fonseca, M. (2006) Genome-wide identification of functionally distinct subsets of cellular mRNAs associated with two nucleocytoplasmic-shuttling mammalian splicing factors. Genome Biol. 7, R113.

77 Bushell, M., Stoneley, M., Kong, Y. W., Hamilton, T. L., Spriggs, K. A., Dobbyn, H. C., Qin, X., Sarnow, P. and Willis, A. E. (2006) Polypyrimidine tract binding protein regulates IRES-mediated gene expression during apoptosis. Mol. Cell 23, 401-412.

78 Maris, C., Dominguez, C. and Allain, F. H. (2005) The RNA recognition motif, a plastic RNA-binding platform to regulate post-transcriptional gene expression. FEBS J. 272, 2118-2131.

79 Conte, M. R., Grune, T., Ghuman, J., Kelly, G., Ladas, A. Matthews, S. and Curry, S. (2000) Structure of tandem RNA recognition motifs from polypyrimidine tract binding protein reveals novel features of the RRM fold. EMBO J. 19,3132-3141.

80 Vitali, F., Henning, A., Oberstrass, F. C., Hargous, Y., Auweter, S. D., Erat, M. and Allain, F. H. (2006) Structure of the two most C-terminal RNA recognition motifs of PTB using segmental isotope labeling. EMBO J. 25, 150-162.

81 Bae, E., Reiter, N. J., Bingman, C. A., Kwan, S. S., Lee, D. Phillips, G. N. Jr., Butcher, S. E. and Brow, D. A. (2007) Structure and interactions of the first three RNA recognition motifs of splicing factor prp24. J. Mol. Biol. 367, 1447-1458

82 Simpson, P. J., Monie, T. P., Szendroi, A., Davydova, N., Tyzack, J. K., Conte, M. R., Read, C. M., Cary, P. D., Svergun, D. I., Konarev, P. V., Curry, S. and Matthews, S. (2004) Structure and RNA interactions of the N-terminal RRM domains of PTB. Structure 12, 1631-1643.

83 Petoukhov, M. V., Monie, T. P., Allain, F. H., Matthews, S. Curry, S. and Svergun, D. I. (2006) Conformation of polypyrimidine tract binding protein in solution. Structure 14, 1021-1027.

84 Monie, T. P., Hernandez, H., Robinson, C. V., Simpson, P., Matthews, S. and Curry, S. (2005) The polypyrimidine tract binding protein is a monomer. RNA 11, 1803-1808.

85 Oberstrass, F. C., Auweter, S. D., Erat, M., Hargous, Y., Henning, A., Wenter, P., Reymond, L., Amir-Ahmady, B., Pitsch, S., Black, D. L. and Allain, F. H. (2005) Structure of PTB bound to RNA: specific binding and implications for splicing regulation. Science 309, 2054-2057.

86 Yuan, X., Davydova, N., Conte, M. R., Curry, S. and Matthews, S. (2002) Chemical shift mapping of RNA interactions with the polypyrimidine tract binding protein. Nucleic Acids Res. 30, 456-462.

87 Auweter, S. D., Oberstrass, F. C. and Allain, F. H. (2006) Sequence-specific binding of single-stranded RNA: is there a code for recognition? Nucleic Acids Res. 34, 4943-4959.

88 Rideau, A. P., Gooding, C., Simpson, P. J., Monie, T. P., Lorenz, M., Huttelmaier, S., Singer, R. H., Matthews, S., Curry, S. and Smith, C. W. (2006) A peptide motif in Raver1 mediates splicing repression by interaction with the PTB RRM2 domain. Nat. Struct. Mol. Biol. 13, 839-848.

89 Sickmier, E. A., Frato, K. E., Shen, H., Paranawithana, S. R., Green, M. R. and Kielkopf, C. L. (2006) Structural basis for polypyrimidine tract recognition by the essential pre-mRNA splicing factor U2AF65. Mol. Cell 23, 49-59.

90 Auweter, S. D., Oberstrass, F. C. and Allain, F. H. (2007) Solving the structure of PTB in complex with pyrimidine tracts: an NMR study of protein-RNA complexes of weak affinities. J. Mol. Biol. 367, 174-186.

91 Schmid, N., Zagrovic, B. and van Gunsteren, W. F. (2007) Mechanism and thermodynamics of binding of the polypyrimidine tract binding protein to RNA. Biochemistry 46, 65006512.

92 Deo, R. C., Bonanno, J. B., Sonenberg, N. and Burley, S. K. (1999) Recognition of polyadenylate RNA by the poly(A)binding protein. Cell 98, 835-845.

93 Handa, N., Nureki, O., Kurimoto, K., Kim, I., Sakamoto, H., Shimura, Y., Muto, Y. and Yokoyama, S. (1999) Structural basis for recognition of the tra mRNA precursor by the sexlethal protein. Nature 398, 579-585.

94 Allain, F. H., Bouvet, P., Dieckmann, T. and Feigon, J. (2000) Molecular basis of sequence-specific recognition of preribosomal RNA by nucleolin. EMBO J. 19, 6870-6881.

95 Kim, S. M. and Jeong, Y. S. (2006) Polypyrimidine tractbinding protein interacts with the 3' stem-loop region of Japanese encephalitis virus negative-strand RNA. Virus Res. 115, 131-140.

96 Maines, T. R., Young, M., Dinh, N. N. and Brinton, M. A. (2005) Two cellular proteins that interact with a stem loop in the simian hemorrhagic fever virus 3'(+)NCR RNA. Virus Res. 109, 109-124.

97 Pilipenko, E. V., Pestova, T. V., Kolupaeva, V. G., Khitrina, E. V., Poperechnaya, A. N., Agol, V. I. and Hellen, C. U. (2000) A cell cycle-dependent protein serves as a templatespecific translation initiation factor. Genes Dev. 14, $2028-$ 2045.

98 Ito, T. and Lai, M. M. (1999) An internal polypyrimidinetract-binding protein-binding site in the hepatitis $\mathrm{C}$ virus RNA attenuates translation, which is relieved by the 3'untranslated sequence. Virology 254, 288-296.

99 Serrano, P., Pulido, M. R., Saiz, M. and Martinez-Salas, E. (2006) The 3' end of the foot-and-mouth disease virus genome establishes two distinct long-range RNA-RNA interactions with the 5' end region. J. Gen. Virol. 87, 3013-3022.

100 Clerte, C. and Hall, K. B. (2006) Characterization of multimeric complexes formed by the human PTB1 protein on RNA. RNA $12,457-475$.

101 He, X., Pool, M., Darcy, K. M., Lim, S. B., Auersperg, N., Coon, J. S. and Beck, W. T. (2007) Knockdown of polypyrimidine tract-binding protein suppresses ovarian tumor cell growth and invasiveness in vitro. Oncogene 26, 4961-4968.

102 Jin, Y., Suzuki, H., Maegawa, S., Endo, H., Sugano, S., Hashimoto, K., Yasuda, K. and Inoue, K. (2003) A vertebrate RNA-binding protein Fox-1 regulates tissue-specific splicing via the pentanucleotide GCAUG. EMBO J. 22, 905-912.

103 Chan, R. C. and Black, D. L. (1997) The polypyrimidine tract binding protein binds upstream of neural cell-specific c-src exon N1 to repress the splicing of the intron downstream. Mol. Cell. Biol. 17, 4667-4676.

104 Lou, H., Helfman, D. M., Gagel, R. F. and Berget, S. M. (1999) Polypyrimidine tract-binding protein positively regulates inclusion of an alternative 3'-terminal exon. Mol. Cell. Biol. 19, 78-85.

\section{To access this journal online: http://www.birkhauser.ch/CMLS}

